\title{
PHOTOMULTIPLIER GAIN TUNING SYSTEM
}

IHEP $^{2}-$ IISN $^{2}-$ LAPP $^{3}$ Collaboration

F. Binon ${ }^{2}$, S.V. Donskov ${ }^{1}$, P. Dutei $1^{4}$, M. Gouanere ${ }^{3}$, V.A. Kachanov ${ }^{1}$, A.V. Kulik ${ }^{1}$, D. Michotte ${ }^{2}$, Yu.V. Mikhailov', J.P. Peigneux ${ }^{3}$, Yu.D. Prokoshkin ${ }^{2}$, A.V. Singovsky ${ }^{1}$ and J.P. Stroot ${ }^{2}$

\author{
Joint Experiment of IHEP Serpukhov, USSR \\ and \\ CERN, Geneva, Switzerland
}

Submitted to Nuclear Instruments and Methods

1 Institute for High Energy Physics, Serpukhov, USSR.

2 Institut Interuniversitaire des Sciences Nucléaires, Bruxelles, Belgium.

3 Laboratoire d'Annecy de Physique des Particules, France.

4 CERN, Geneva, Switzerland. 


\section{INTRODUCTION}

Most high energy physics experiments today make use of large number of photomultipliers (PM). The setting and the tuning of the high voltage on the photomultiplier dividers need automatic devices. A few of these have been developed and work satisfactorily. Nevertheless this note describes a highly reliable system which is definitely cheaper to build and to maintain in most cases and which is particularly suited for large calorimeters with thousands PM.

This system has been developed to feed high voltage on the photomultipliers of a lead glass counter matrix, the electromagnetic calorimeter GAMS [1,2] (at IHEP the dimensions of the matrix are $32 \times 48$. i.e. 1536 PM; at CERN - NA12 experiment - they are $64 \times 64$, i.e. 4096 PM).

The problem with calorimeters is not so much to control the stability of the power supply and to keep constant fixed high voltage values on each PM as to control the gain of each PM. There is no univocal relation between gain and high voltages in a photomultipliex. Gain hysteresis results after prolonged illumination of the photocathode and of the dynodes or vith large variations in light intensity. The gain may also vary substantially over the duration of each burst in high intensity pulsed particle beams. These short time drifts cannot be controlled by high voltage regulation in a practical way on a large scale. They can only be monitored $[1,3]$.

With these conditions a PM HV regulation system should:

(a) make the initial tuning of the high voltage on each individual P.M. so as to equalise all gains with the help of a gain measuring device in the case of a calorimeter;

(b) allow to bring the necessary corrections to long term drifts at given intervals or requests.

Such a system must have enough flexibility and be computer (and/or manually) controlled. The survey functions are left to the gain measurement set-up for calorimeters. For ordinary scintillation counters, provision is made for a high voltage measurement. 
In any case, high voltage changes are quite unfrequent except during the setting up of an experiment. So the system does not need to be particularly fast. Cheap and reliable small low voltage (typically 4V) d.c. motors that are available nowadays may be used to drive the axis of small variable resistors for high voltage tuning. They have the advantage over electronics controls that settings are not disturbed by any power failure in the system [4] and even can be manually adjusted in case of need.

The system which is used for GAMS -4000 is described in section 2 . A system for scintillation counters is described in section 3 .

\section{SYSTEM FOR THE CALORIMETER GAMS}

GAMS photomultipliers are the venetian blind 12-stages USSR made FEU 84-3. High voltage dividers have been designed in order to obtain a maximum range of linearity. The last five dynodes are supplied with high current floating power supplies capable of delivering the necessary current during the beam spill without altering high voltage values. These boosting voltages are the same for al1 $4096 \mathrm{PM}$. On the other hand, the mean current through the resistor chain is kept at a low value ( $120 \mu \mathrm{A}$ for a mean HV of $1300 \mathrm{v}$ ). This has the double advantage of keeping to a minimum the dissipated power inside GAMS and of reducing the dimensions of the power packs (Fig. I).

The gain of each PM is adjusted with a $0.75 \mathrm{~W}$ multiturn adjustable $2 M \Omega$ potentiometer in series with the divider. On each divider a d.c. low inertia micromotor ${ }^{*}$ ) with a proper reductor drives the variable resistor $^{* *}$ ) (Fig. 2). The speed of resistance variation has been chosen in order to be able to adjust the PM gain to a few $\%$ easily. The range of adjustment is about a factor five. A fixed value resistor may be inserted in the divider circuit to obtain a fixed HV drop in order to cover a twice larger tuning range. The whole range may be covered in about $30 \mathrm{sec}$.

\footnotetext{
*) Minimotor S.A. Type 1516E 0045 Reduction ratio $485 / 1$ - Voltage $4.5 \mathrm{~V}$ $* *$ ) CERMET - Bourns. Type 3006P Thermal stability: $100 \mathrm{ppm} /{ }^{\circ} \mathrm{C}$.
} 
The matrix geometry of GAMS has influenced the layout of the control and supply of the micromotors. Flat cables connect in parallel 64 PM of a column (Figs. 3,4). Horizontal lines select individual motors. One CAMAC unit may control a relay box for 2048 motors. 32 photomultipliers may be adjusted simultaneously (Figs. 5,6 ).

Monitoring light pulses of $20 \mathrm{~ns}$ duration are fed to each PM [3]. In practice, the gains of all 4096 PM are initially measured in a monochromatic electron beam (GAMS may be moved laterally in both horizontal and vertical directions). The corresponding $A D C$ values obtained with the light pulse system are then used to control the adjustment of the motors in order to equalise the gains. Equalisation within $\pm 5 \%$ is usually obtained after three computer controlled iterations in about two hours time.

Gains are monitored with the pulsed light system during data taking at each accelerator burst. They may occasionally be readjusted after large drifts (which usually signal a "sick" or defective component) or in the case of PM replacement. In any case the motor system is powered very occasionally only.

\section{SCINTILLATION COUNTER SYSTEMS}

Other geometries may be used depending on the set-up configuration. But the same HV tuning principle has been found convenient even for isolated PM or groups of scintillation counters which may be associated with beam detection, small hodoscopes, etc.

A distribution box capable of driving 16 or 32 PM has been designed and is being used for all beam counters in the CERN NA12 experiment (Fig. 7). HV measurement is provided with a CAMAC multichannel DVM. In this way, plateau curves may be measured under computer program control.

\section{CONCLUSION}

A reliable computer controlled HV tuning system for medium and large numbers of PM has been described. It is cheap and easy to handle. Being electrically disconnected except at tuning time, it is not susceptible to noise and it is not perturbed by electric failure. Such a system has been in use during the last three years both at IHEP and at CERN. 


\section{REFERENCES}

[1] F. Binon et al., Nuc1. Inst. Mech. 188 (1981) 507.

[2] V. Davydov et al., Lett. Nuovo Cimento 32 (1981) 45.

[3] F. Binon et al., (to be published).

[4] P. Duteil et al., CERN/77-11.

\section{FIGURE CAPTIONS}

Fig. 1 Schematics of the high voltage divider for FEU 84-3.

Fig. 2 Assembly of a PM and its divider.

Fig. 3 Schematics of the gain control set-up for GAMS.

Fig. 4 Connection layout for GAMS-like (matrix) geometry.

Fig. 5 Control electronics block diagram.

Fig. 6 Motor drivers.

Fig. 732 PM H.V. distribution box. 


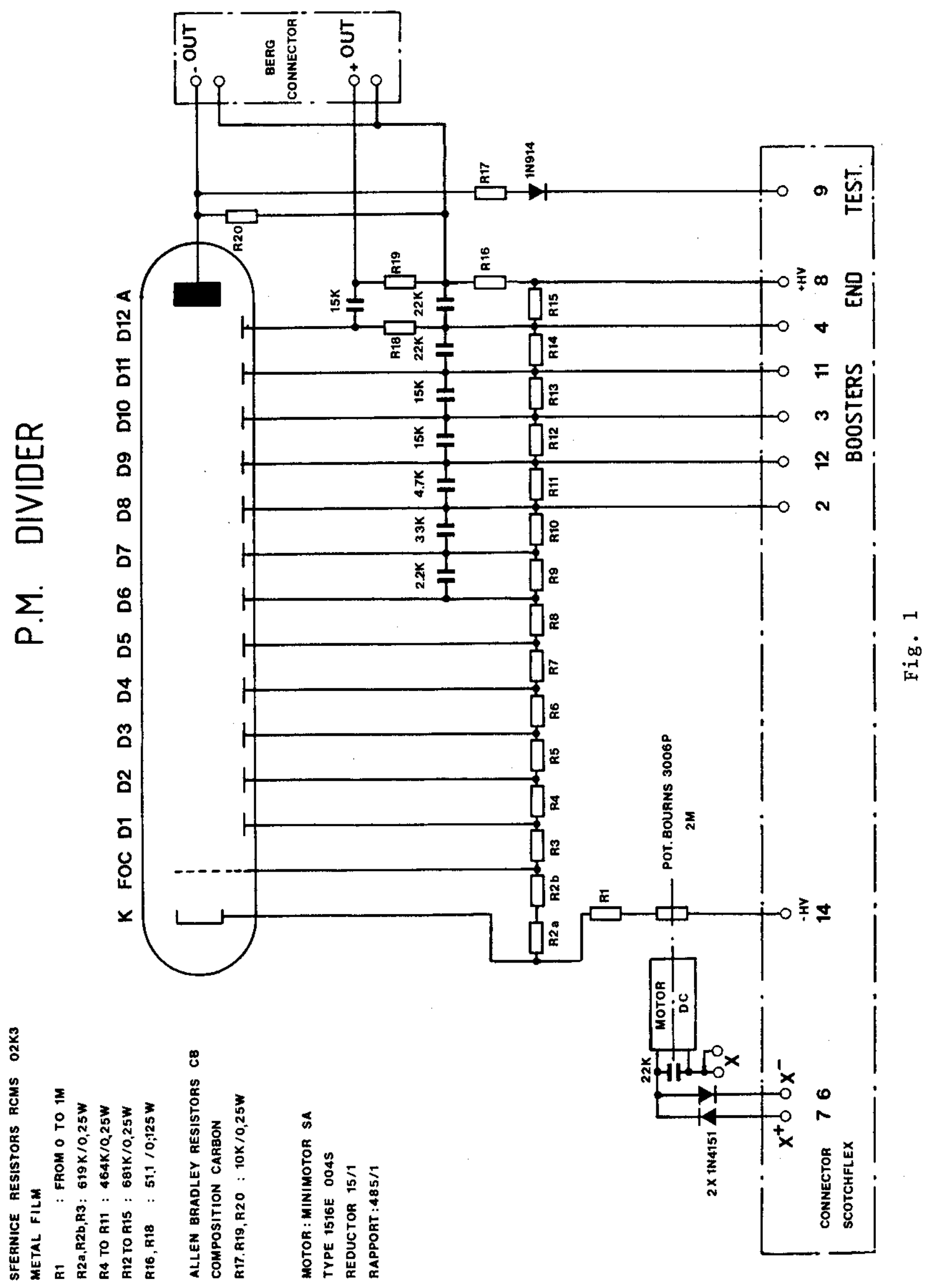




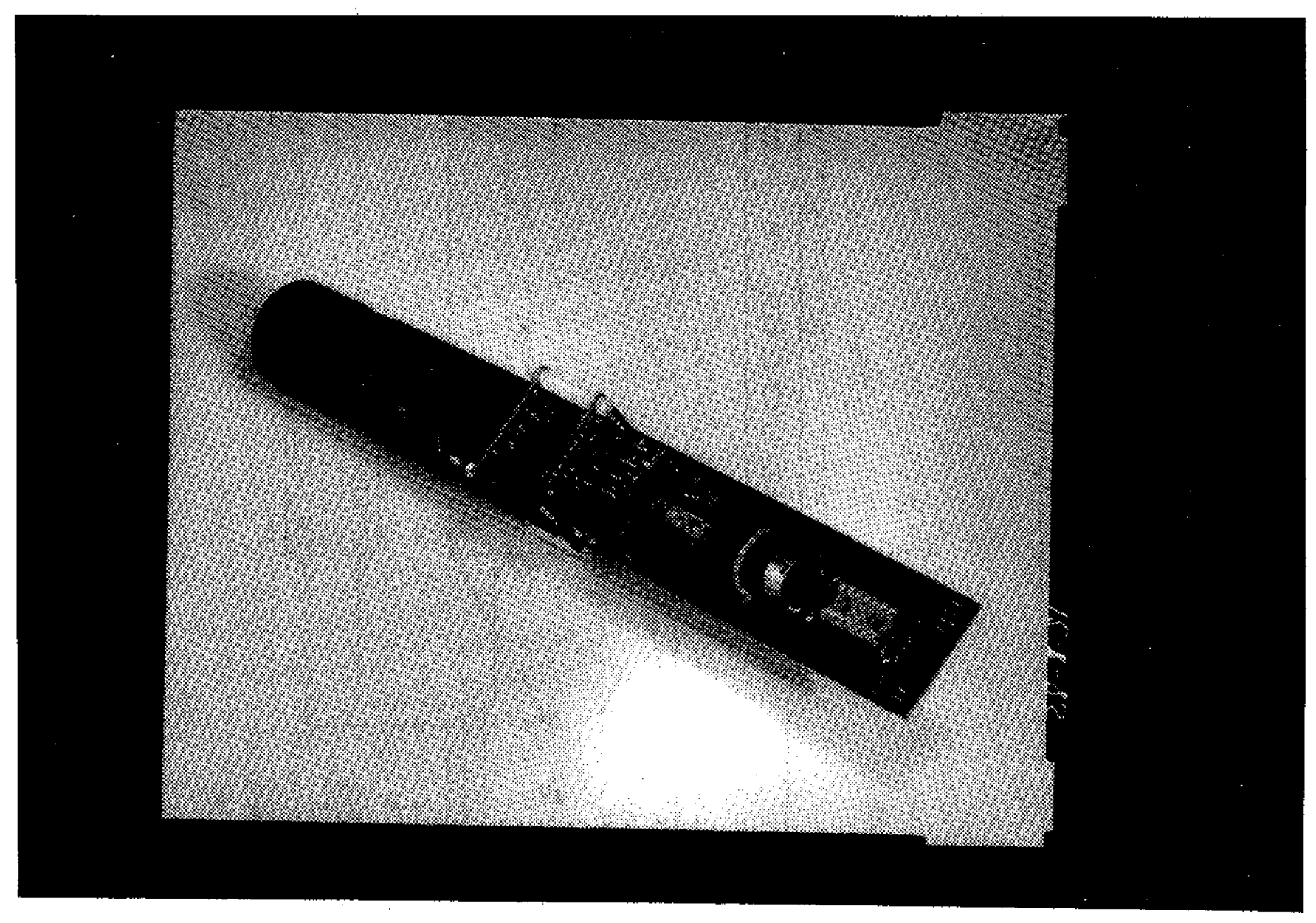

Fig. 2 


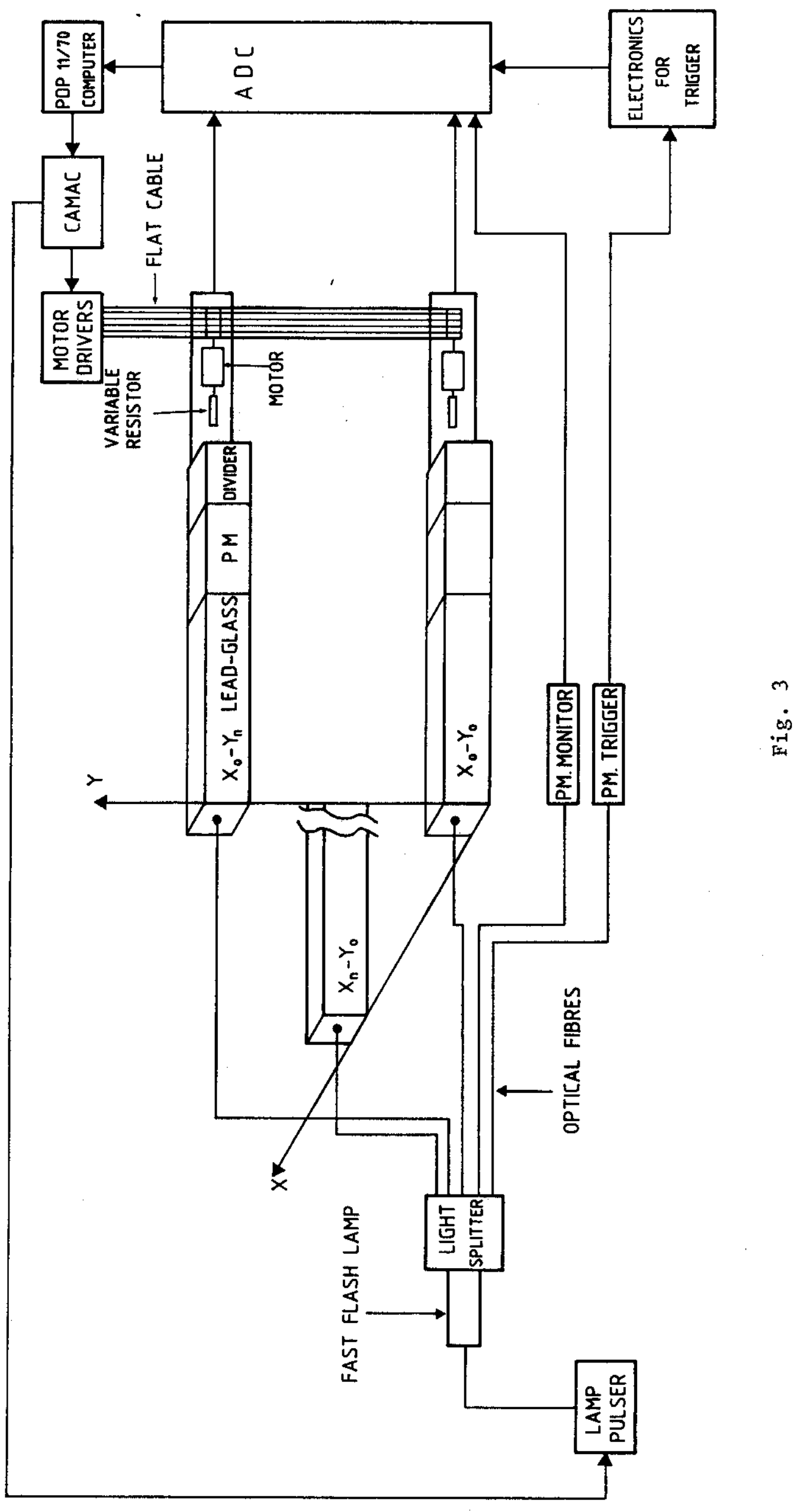




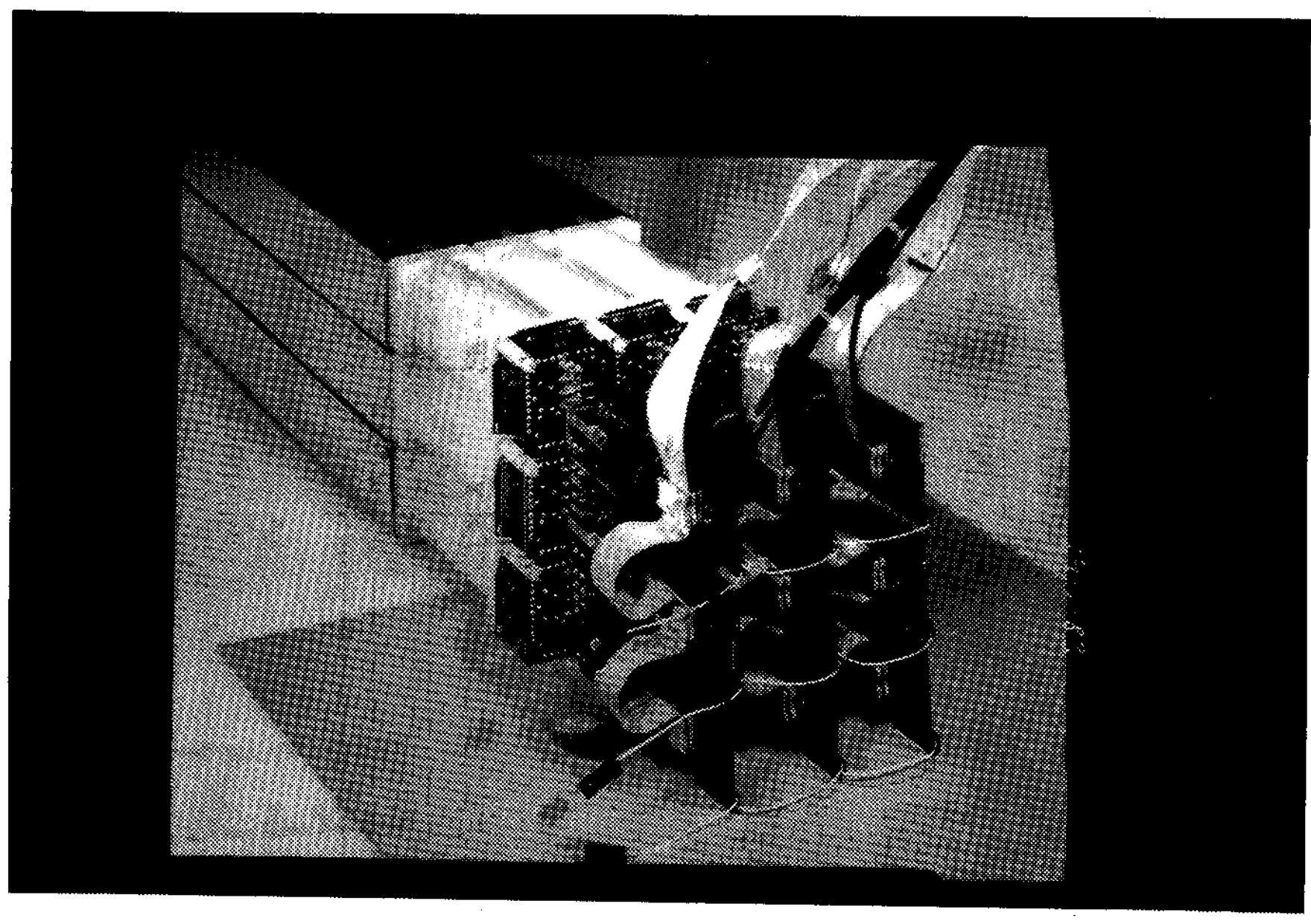

Fig. 4 


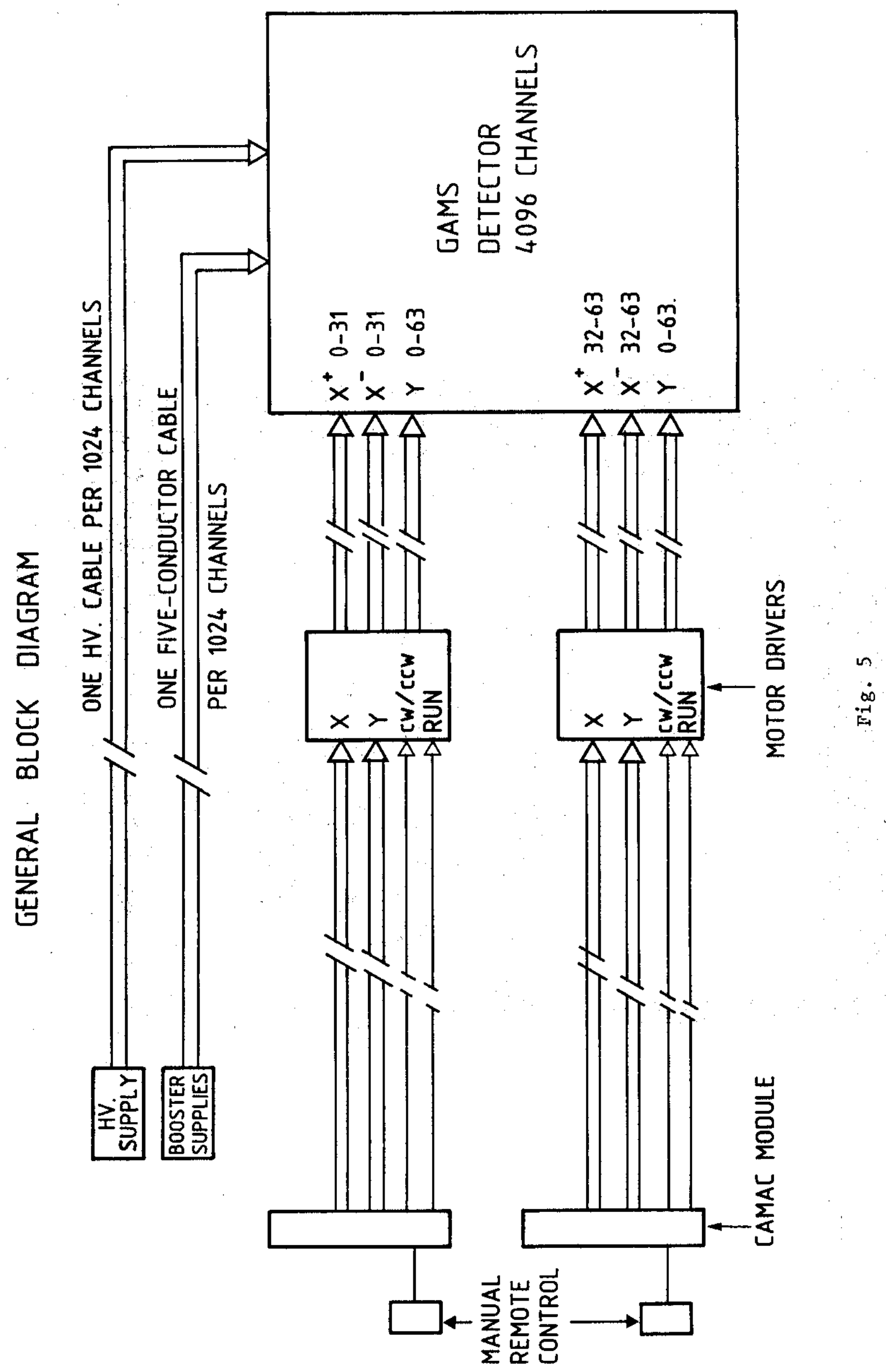




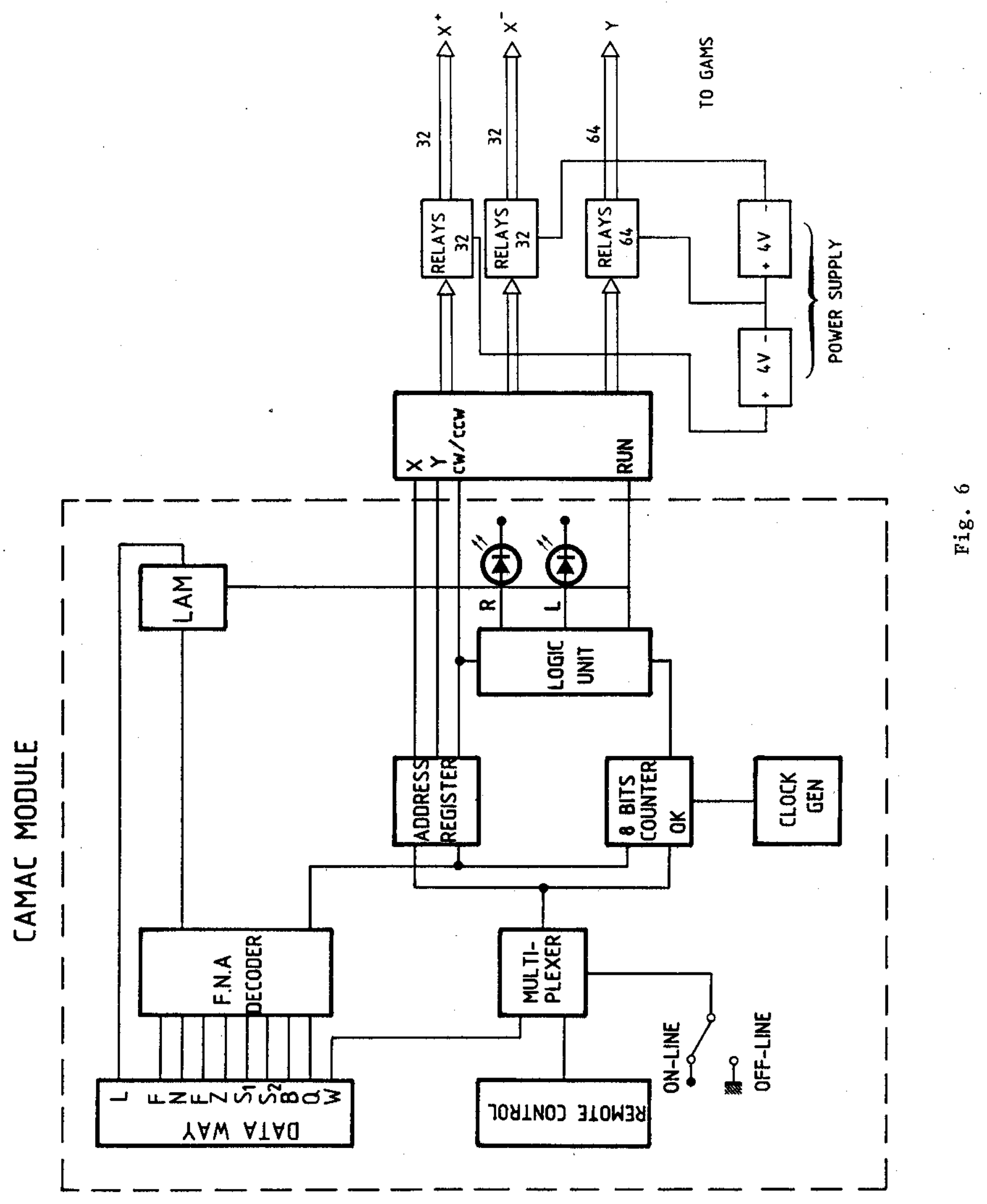




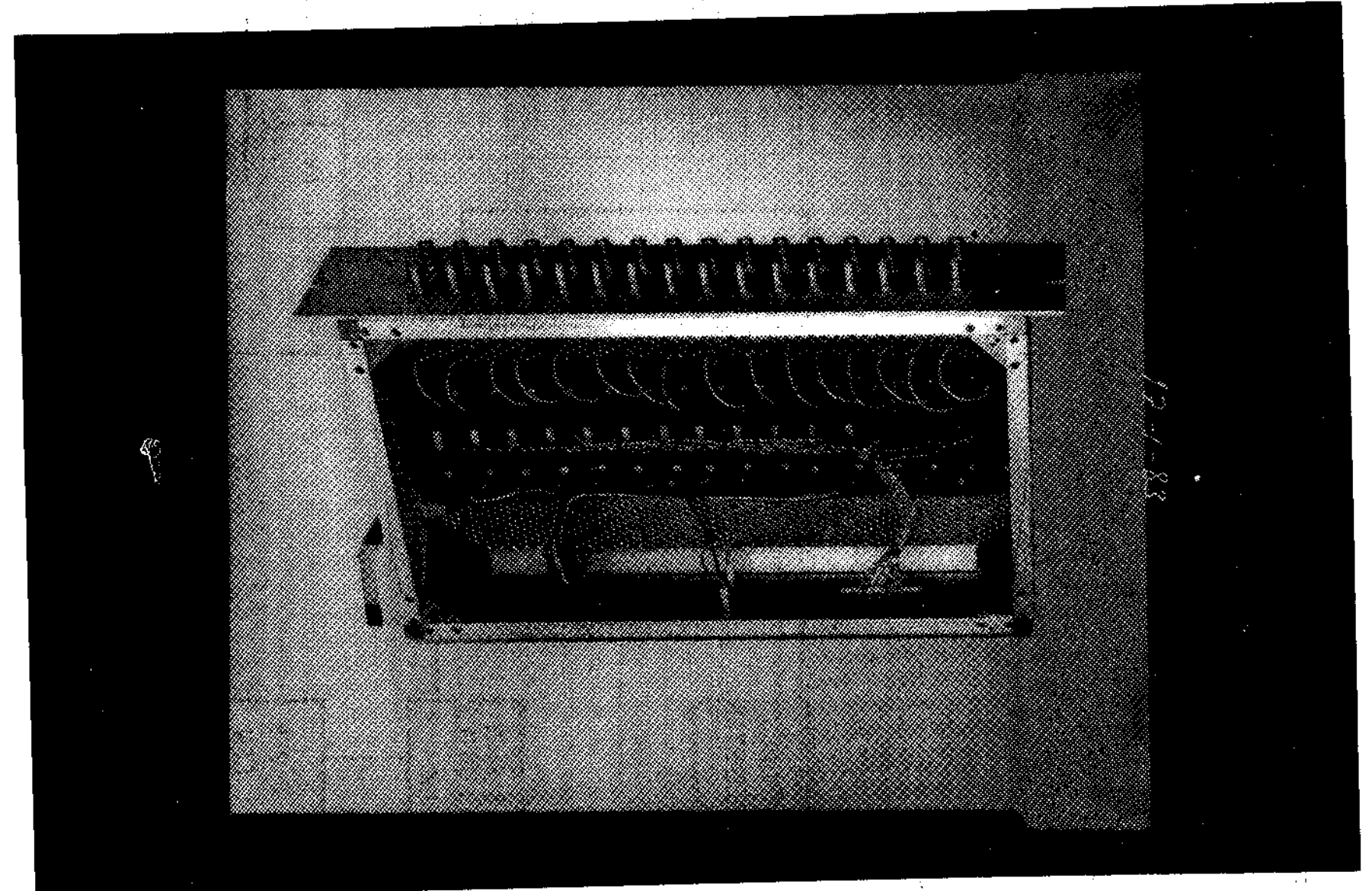

Fig. 7 